\title{
Compact Ultra-Wideband Bandpass Filters Using Composite Microstrip-Coplanar-Waveguide Structure
}

\author{
Tsung-Nan Kuo, Shih-Cheng Lin, and Chun Hsiung Chen, Fellow, IEEE
}

\begin{abstract}
Compact ultra-wideband bandpass filters are proposed based on the composite microstrip-coplanar-waveguide (CPW) structure. In this study, the microstrip-CPW transitions and the CPW shorted stubs are adopted as quasi-lumped-circuit elements for realizing a three-pole high-pass filter prototype. By introducing a cross-coupled capacitance between input and output ports of this high-pass filter and suitably designing the transition stretch stubs, a compact three-pole ultra-wideband bandpass filter is implemented with two transmission zeros located close to the passband edges. To further improve the selectivity, two microstrip shorted stubs are added to implement a five-pole ultra-wideband bandpass filter with good out-of-band response. Being developed from the quasi-lumped elements, and not from the transmission lines, the proposed ultra-wideband filters have sizes more compact than those of the published wideband filters. The proposed ultrawideband filters have the merits of compact size, flat group delay, good insertion/return loss, and good selectivity. Agreement between simulated and measured responses of these filters is demonstrated.
\end{abstract}

Index Terms-Bandpass filter, coplanar waveguide (CPW), cross coupling, high-pass filter, microstrip, ultra-wideband (UWB).

\section{INTRODUCTION}

$\mathbf{U}$ LTRA-WIDEBAND (UWB) radio technology has been getting more and more popular for high-speed wireless connectivity, since the Federal Communications Commission (FCC)'s decision to permit the unlicensed operation band from 3.1 to $10.6 \mathrm{GHz}$ in February 2002 [1]. There are several advantages for UWB radio system, such as transmitting higher data rates, needing lower transmit power, and simplifying the error control coding. In such a system, an UWB filter is one of the key components, which should exhibit a wide bandwidth with low insertion loss over the whole band. In order to meet the FCC limit, good selectivity at both lower and higher frequency ends and flat group-delay response over the whole band are required.

The conventional filter synthesis procedure is adequate only for the relatively narrowband filters, and is not suitable for the wideband filters [2]. Basically, a wide bandwidth filter may be implemented by a direct cascade of the low- and high-pass filters. In [3], the wideband coplanar-waveguide (CPW) bandpass filters based on the cascade of CPW low- and high-pass periodic structures were constructed. To save the circuit area, the

Manuscript received February 13, 2006. This work was supported by the National Science Council of Taiwan under Grant NSC-94-2752-E-002-001-PAE, Grant NSC-94-2213-E-002-055, and Grant NSC-94-2219-E-002-008.

The authors are with the Department of Electrical Engineering and Graduate Institute of Communication Engineering, National Taiwan University, Taipei 106, Taiwan, R.O.C. (e-mail: chchen@ew.ee.ntu.edu.tw).

Digital Object Identifier 10.1109/TMTT.2006.881624 wideband bandpass filters were proposed by combining the lowand high-pass filters together [4]. These wideband filters have good suppression on out-of-band response, however, they have the drawback of large circuit size or imperfect group delay over the passband.

To meet the UWB filter specification, several studies to increase the number of sections have been reported. In [5], the high-pass filter consisting of a cascade of shunt short-circuited stubs was adopted for the UWB filter design. In [6], the UWB filter was constructed by a cascade of various ring filters. These UWB filters have sharp rejection, but their spurious response would degrade the out-of-band response and an increase in number of sections may lead to large insertion loss, as well as poor group delay.

Another way to realize wideband bandpass filters is to use the parallel-coupled lines [7], [8]. In [9], the parallel-coupled microstrip line with defected ground was employed to give a tight coupling for a wideband bandpass filter. However, the more the fractional bandwidth is required, the smaller the gap size is demanded to enhance the coupling. In [10], by adding a third line in the parallel coupled line design, the restriction on gap size may be relaxed for a specific coupling, but for UWB filter design, the necessary gap size is still too narrow to be fabricated.

Recently, broadside-coupled structures have received great attention due to the merit associated with the electromagnetic coupling. The CPW-to-CPW transitions were first proposed in [11], accompanied by the development of CPW-to-microstrip transitions [12] and microstrip-to-CPW transitions [13]. In [14], the UWB filter with a multiple-mode resonator was proposed using the microstrip-to-CPW transitions as inverter circuits. Recently, a UWB filter was proposed using the microstrip-to-CPW transitions to realize the broadside-coupled structure and then cascading three sections to give good selectivity [15].

In this paper, compact UWB bandpass filters are developed by adopting the high-pass filter prototype and transition stretch stubs to create the lower and upper stopbands, respectively. Specifically, a composite microstrip-CPW structure is proposed to realize a lumped-element high-pass filter prototype, which is essential in developing the UWB bandpass filters. First, by using the microstrip-to-CPW transition structures to implement two series lumped capacitors, using a CPW shorted stub structure to implement the shunt inductor, and also introducing a suitable cross-coupled capacitor between the input and output ports, a very wideband three-pole bandpass filter is developed from the corresponding three-pole high-pass filter prototype. Second, by suitably modifying the shape of 


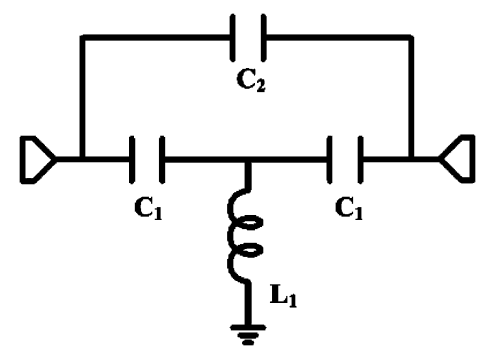

Fig. 1. Circuit diagram of the three-pole high-pass filter prototype.

microstrip-to-CPW transition to create the microstrip stretch stub for adjusting the upper transmission zero, a three-pole UWB bandpass filter is fabricated with two transmission zeros properly located. Finally, to give more sharp rejection around the upper stopband, two microstrip shorted stubs are incorporated into the three-pole UWB bandpass filter so that a five-pole UWB bandpass filter is implemented. Being that the quasi-lumped elements in the filter design is adopted, the proposed UWB filters exhibit the slow wave behavior and have sizes more compact than those of published wideband filters.

\section{High-PASS-BASED BANDPASS FILTER}

Fig. 1 shows the lumped-element three-pole high-pass filter prototype that will be adopted to develop a very wideband bandpass filter. Here, the series capacitances $C_{1}$ and shunt inductance $L_{1}$ are selected to decide the cutoff frequency of the highpass filter, and the cross-coupled capacitance $C_{2}$ is introduced to create a transmission zero at the passband edge so that the filter selectivity at the lower stopband may be improved. Fig. 2(a) shows the circuit simulated frequency response of this threepole high-pass filter prototype for which the value of cross-coupled capacitance $C_{2}$ is adjusted to control the location of the created transmission zero.

The even- and odd-mode analysis technique can be used to discuss the transmission zero [16]. By symmetry of the circuit model in Fig. 1, the even- and odd-mode input impedances ( $Z_{\text {even }}$ and $Z_{\text {odd }}$ ) are expressed as

$$
\begin{aligned}
& Z_{\text {even }}=j\left(2 w L_{1}-\frac{1}{w C_{1}}\right) \\
& Z_{\text {odd }}=\frac{1}{j w\left(C_{1}+2 C_{2}\right)} .
\end{aligned}
$$

By superposition, the transfer function $S_{21}$ is written as

$$
S_{21}=\frac{\left(Z_{\text {even }}-Z_{\text {odd }}\right) Z_{0}}{\left(Z_{\text {even }}+Z_{0}\right)\left(Z_{\text {odd }}+Z_{0}\right)}
$$

where $Z_{0}$ is the impedance of the input port. A transmission zero is created when the transfer function $S_{21}$ is equal to zero, i.e.,

$$
Z_{\text {even }}=Z_{\text {odd }} \text {. }
$$

By using (4), the location of transmission zero may be predicted. Fig. 2(b) shows the curve to relate the transmission-zero fre-

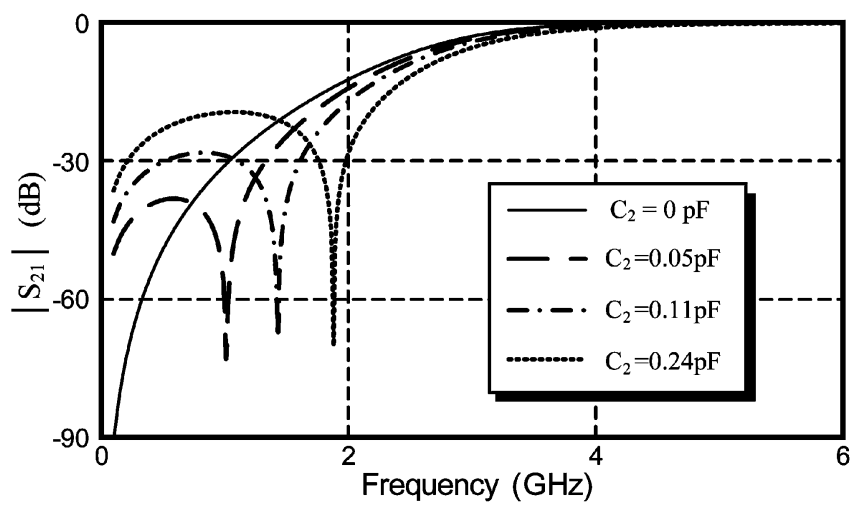

(a)

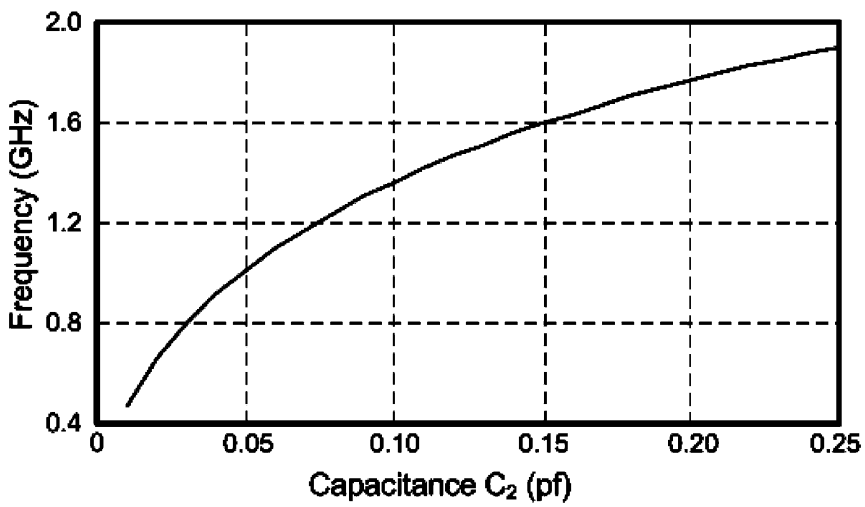

(b)

Fig. 2. (a) Circuit simulated results of the three-pole high-pass filter prototype (Fig. 1) for various values of the cross-coupled capacitance $C_{2}$. (b) Curve to relate the transmission-zero frequency to the value of the capacitance $C_{2} .\left(C_{1}=\right.$ $\left.0.858 \mathrm{pF}, L_{1}=1.5 \mathrm{nH}\right)$.

quency to the value of cross-coupled capacitance $C_{2}$ under the case of $C_{1}=0.858 \mathrm{pF}$ and $L_{1}=1.5 \mathrm{nH}$. As the value of $C_{2}$ increases, the transmission zero will move toward the higher frequency.

To realize the circuit model in Fig. 1, the series capacitors $C_{1}$ are implemented by the microstrip-to-CPW transitions, and the shunt inductor $L_{1}$ is implemented by the CPW shorted stub connected to the ground. The cross-coupled capacitor $C_{2}$ is formed between the adjacent microstrip parts of the two capacitors $C_{1}$. Fig. 3(a) and (b) shows the three-dimensional and top-/bottomlayer circuit layouts of the proposed filter, respectively, which is developed from the high-pass filter prototype shown in Fig. 1.

The full-wave simulated results of Fig. 3 are shown in Fig. 4, which are calculated by the ADS Momentum simulator and simulated on the substrate with $\varepsilon_{r}=3.38, \tan \delta=0.002$, and thickness $h=0.508 \mathrm{~mm}$. Although the circuit layout (Fig. 3) is developed from the high-pass filter prototype (Fig. 1), the implemented structure (Fig. 3) actually behaves like a wideband bandpass filter for which a stopband starts to appear at $17 \mathrm{GHz}$. Basically, this implemented filter has a very wideband with a 3-dB fractional bandwidth of $134 \%$ from 3.07 to $15.7 \mathrm{GHz}$ and two transmission zeros are found at 1.88 and $16.81 \mathrm{GHz}$. Note that the first transmission zero is created by the cross-coupled capacitance $C_{2}$ and the second transmission zero is generated by the resonance associated with the microstrip parts of the capacitors $C_{2}$ and the CPW shorted stub. The filter has a min- 


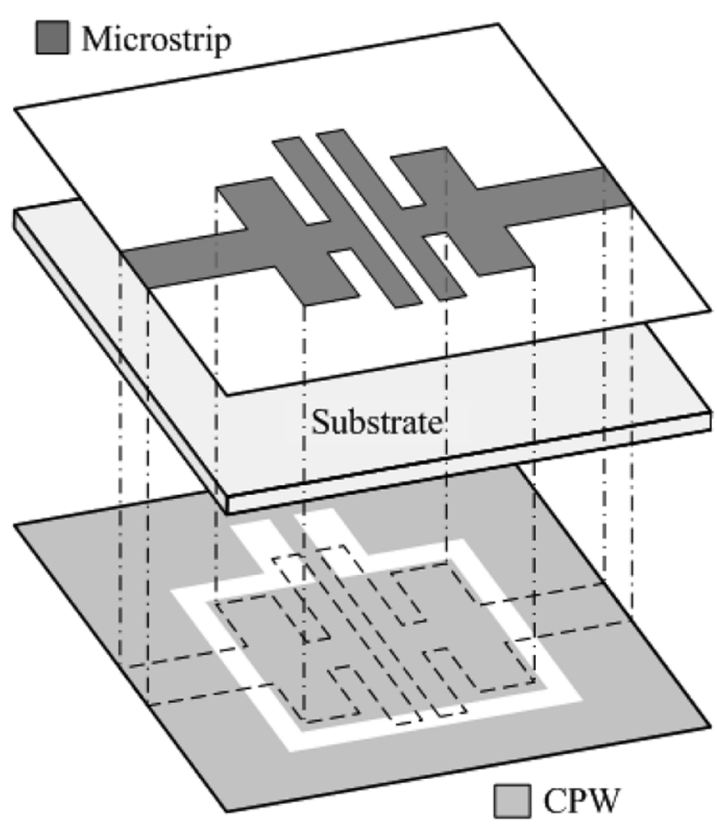

(a)

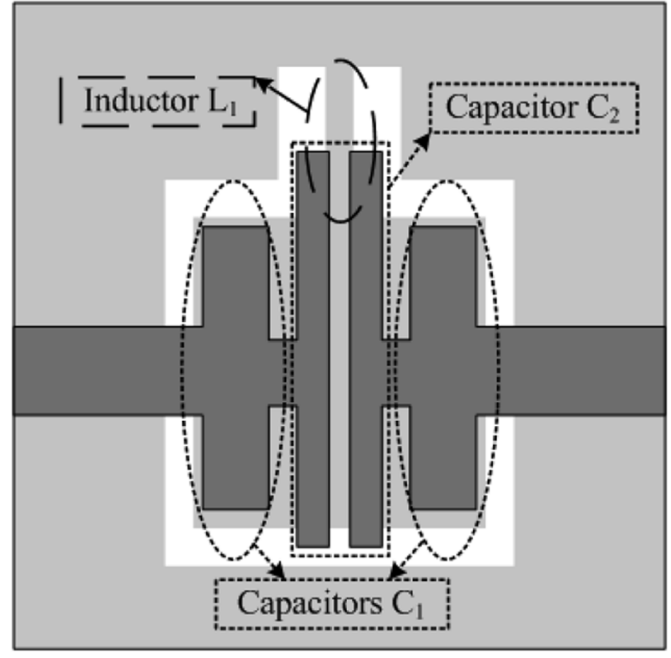

(b)

Fig. 3. (a) Three-dimensional circuit layout of proposed wideband filter. (b) Top-/bottom-layer circuit layouts to show the relative location between top microstrip and bottom CPW layers of proposed wideband filter.

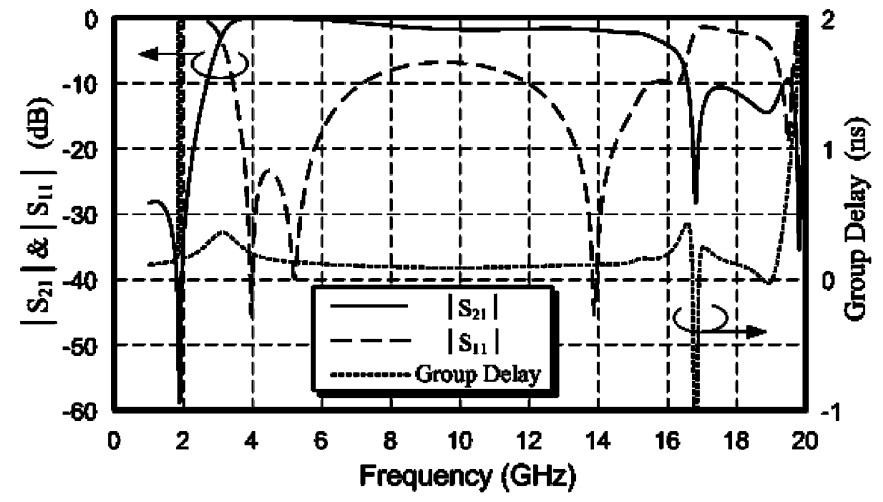

Fig. 4. Simulated results of the proposed filter in Fig. 3.

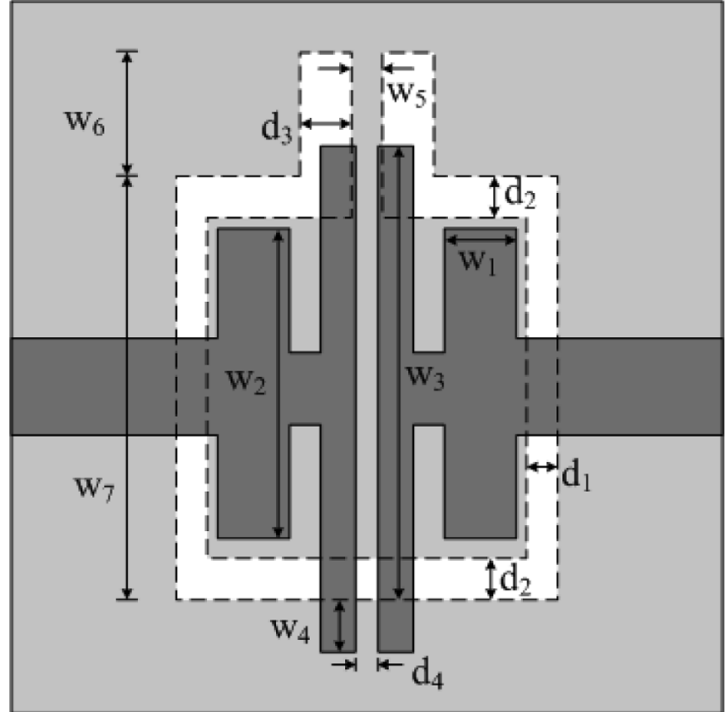

Fig. 5. Top-/bottom-layer circuit layouts of proposed three-pole UWB bandpass filter. $\left(w_{1}=0.89 \mathrm{~mm}, w_{2}=3.81 \mathrm{~mm}, w_{3}=5.59 \mathrm{~mm}, w_{4}=\right.$ $0.635 \mathrm{~mm}, w_{5}=0.38 \mathrm{~mm}, w_{6}=1.52 \mathrm{~mm}, w_{7}=5.21 \mathrm{~mm}, d_{1}=0.38 \mathrm{~mm}$, $d_{2}=0.5 \mathrm{~mm}, d_{3}=0.635 \mathrm{~mm}$, and $d_{3}=0.28 \mathrm{~mm}$ ).

imum insertion loss of $0.1 \mathrm{~dB}$, and the return loss is greater than $6.8 \mathrm{~dB}$ within the passband. Moreover, this filter has a compact dimension of $4.7 \mathrm{~mm} \times 6.73 \mathrm{~mm}$, which is approximately $0.243 \lambda \times 0.347 \lambda$, and $\lambda$ is the guided wavelength of the microstrip structure at the center frequency $f_{0}=9.32 \mathrm{GHz}$. In addition, this wideband bandpass filter exhibits a flat group-delay response below $0.35 \mathrm{~ns}$ over the whole passband.

\section{Three-Pole UWB BANDPASS FILTER}

In Section II, a very wideband bandpass filter is constructed from the three-pole high-pass filter prototype. In order to develop a filter meeting the UWB standard, the high-frequency response needs to decay early. To this end, the microstrip-to-CPW transition structure in Fig. 3 is modified such that part of the microstrip segment is extended over the ground region of microstrip to form a transition stretch stub of length $w_{4}$, as shown in Fig. 5. By this modification, a three-pole UWB bandpass filter (shown in Fig. 5) is developed.

For the proposed three-pole UWB bandpass filter with the circuit layout shown in Fig. 5, the microstrip-to-CPW transitions are used to realize the series capacitances $C_{1}$ and the CPW shorted stub connected to ground is used to realize the shunt inductance $L_{1}$. The adjacent microstrip parts between two transitions are used to implement the cross-coupled capacitance $C_{2}$. Note that the adjacent microstrip portions of two transitions have parts of microstrip segments, the transition stretch stubs, extending over the ground. These stretch stubs are used to generate the transmission zero at the upper passband edge so that the selectivity of the UWB bandpass filter around the upper stopband may be improved.

Fig. 6(a) illustrates the effect of adjusting the length $w_{4}$ of the transition stretch stub covering the ground. It shows that an additional transmission zero will be observed at the higher frequency. Specifically, the upper transmission zero will move to 


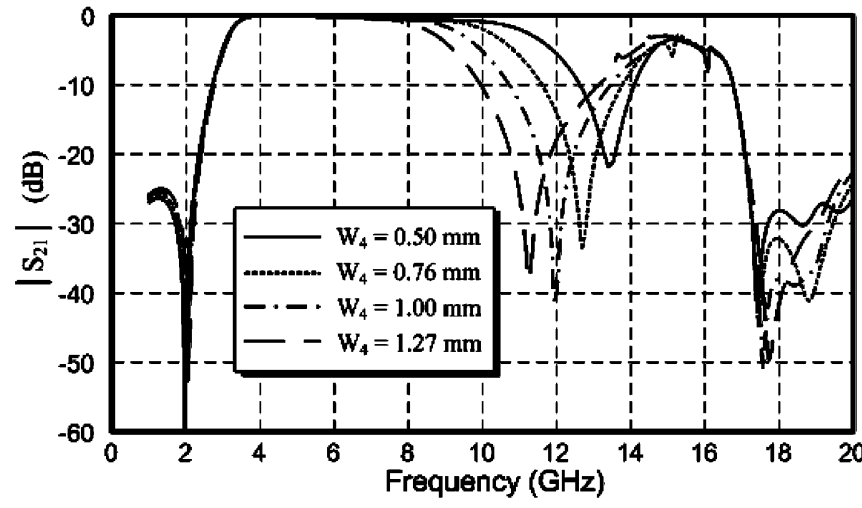

(a)

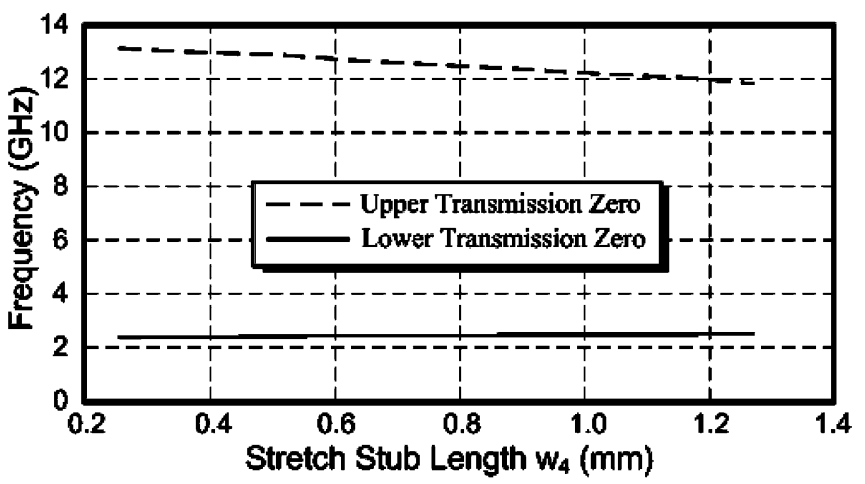

(b)

Fig. 6. (a) Simulated results of the proposed three-pole UWB filter (Fig. 5) for various values of the stretch stub length $w_{4}$. (b) Curves to relate the transmission-zero frequencies to the values of the stretch stub length $w_{4}$.

the lower frequency as the stretch stub length $w_{4}$ is increased. Note that an adjustment of the stretch stub length $w_{4}$ also has a minor effect on the cross-coupled capacitance $C_{2}$ with the lower transmission zero moving slightly to the high frequency as $w_{4}$ is increased. The transmission-zero frequency versus the stretch stub length $w_{4}$ is plotted in Fig. 6(b), where the upper transmission zero decreases almost linearly and the lower transmission zero increases slightly when the length $w_{4}$ is increased. By designing a three-pole high-pass filter and introducing a suitable stretch stub, a compact three-pole UWB bandpass filter is implemented with two transmission zeros located close to the passband edges.

Fig. 7(a) shows the measured and full-wave simulated responses of the three-pole UWB bandpass filter (Fig. 5), which is fabricated on the Rogers RO4003C substrate with $\varepsilon_{r}=3.38$, $\tan \delta=0.002$, and thickness $h=0.508 \mathrm{~mm}$. The measured center frequency is at $6.8 \mathrm{GHz}$ and the measured $3-\mathrm{dB}$ fractional bandwidth is $109.7 \%$ from 3.07 to $10.53 \mathrm{GHz}$. This implemented filter has a minimum insertion loss of $0.4 \mathrm{~dB}$, and the return loss is greater than $11 \mathrm{~dB}$ within the passband. Two transmission zeros are found at 1.919 and $13.07 \mathrm{GHz}$. The implemented filter with the input and output feeding transmission lines deembedded presents a flat group-delay response below $0.36 \mathrm{~ns}$ over the whole passband. Note that the group delay exhibits negative numbers around the frequencies at which the transmission zeros are observed, as shown in Fig. 7(b). Besides, the proposed three-pole UWB bandpass filter has a very

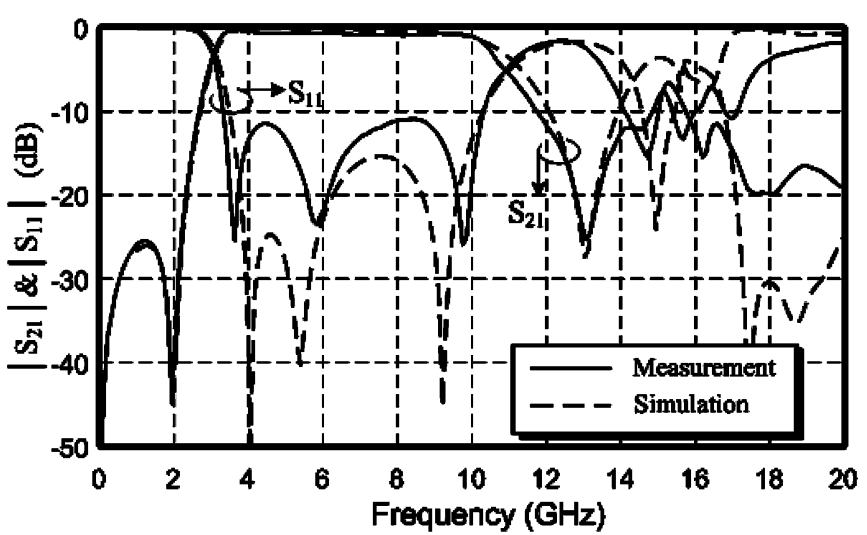

(a)

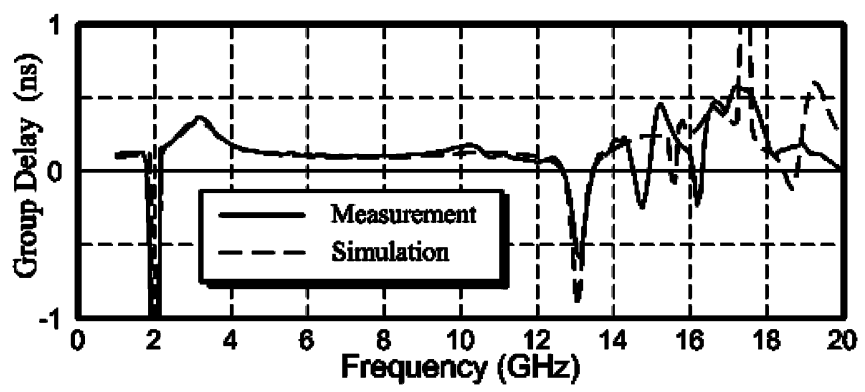

(b)

Fig. 7. Measured and simulated results of the proposed three-pole UWB filter (Fig. 5). (a) Scattering parameters. (b) Group delay.

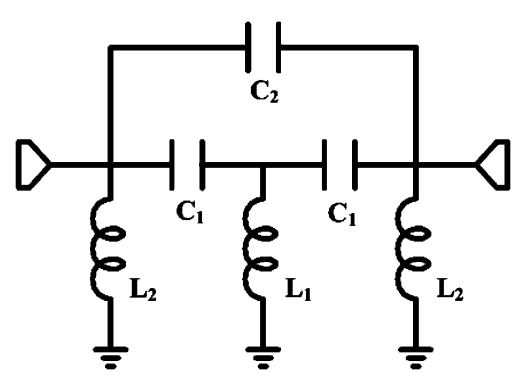

Fig. 8. Circuit diagram of the five-pole high-pass filter prototype.

compact size of $4.7 \mathrm{~mm} \times 6.73 \mathrm{~mm}$, which is approximately $0.177 \lambda \times 0.253 \lambda$ and where $\lambda$ is the guided wavelength of microstrip structure at the center frequency $f_{0}=6.8 \mathrm{GHz}$.

\section{Five-Pole UWB BandPass FILTER}

Although the three-pole UWB bandpass filter (Fig. 5) shows good performance and compact size, it still cannot meet the FCC's limit due to its poor selectivity at higher frequency. To improve the selectivity and to have more attenuation in the stopband, one may modify the three-pole UWB bandpass filter structure in Fig. 5 to form a new five-pole UWB bandpass filter, which is also developed from a five-pole high-pass filter prototype.

Shown in Fig. 8 is the circuit model of a five-pole high-pass filter prototype, as discussed in [2]. By inserting a cross-coupled capacitance $C_{2}$ between the input and output ports of the five-pole high-pass filter prototype, a transmission zero can be created at the lower stopband, as shown in Fig. 9(a). Due to the 


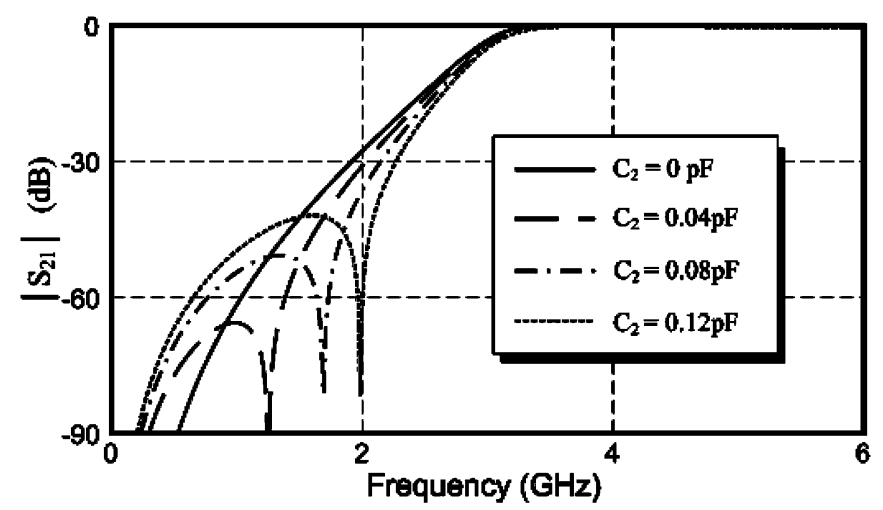

(a)

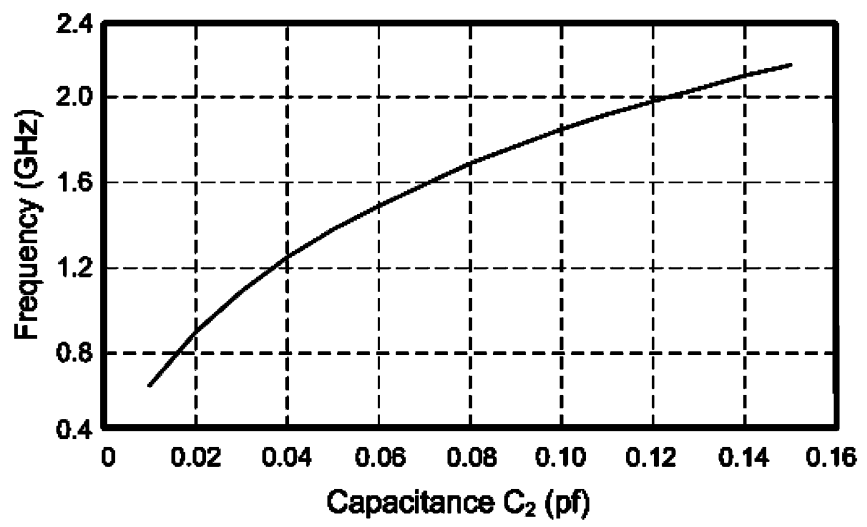

(b)

Fig. 9. (a) Circuit simulated results of the five-pole high-pass filter prototype (Fig. 8) for various values of the cross-coupled capacitance $C_{2}$. (b) Curve to relate the transmission-zero frequency to the value of the capacitance $C_{2} .\left(L_{1}=\right.$ $\left.1 \mathrm{nH}, C_{1}=0.768 \mathrm{pF}, L_{2}=1.8 \mathrm{nH}\right)$.

symmetry of the circuit diagram (see Fig. 8), the transmission zero may also be discussed by the even- and odd-mode analysis technique. For the case of $L_{1}=1 \mathrm{nH}, C_{1}=0.768 \mathrm{pF}$, and $L_{2}=1.8 \mathrm{nH}$, the curve to relate the transmission-zero frequency to the value of the cross-coupled capacitance $C_{2}$ is plotted in Fig. 9(b). Note that the transmission zero will move toward the higher frequency as the value of $C_{2}$ increases.

Fig. 10 shows the circuit layout of the proposed five-pole UWB bandpass filter. This implemented filter is based on the five-pole high-pass filter prototype in Fig. 8. Again, the series capacitors $C_{1}$ are implemented by the microstrip-to-CPW transitions and the shunt inductor $L_{1}$ is implemented by a CPW shorted stub connected to the ground. The cross-coupled capacitance $C_{2}$ is realized by the adjacent microstrip parts of the two capacitors $C_{1}$. The shunt inductors $L_{2}$ are implemented by the microstrip shorted stubs with metal vias to the ground and the metal via has a diameter of $1 \mathrm{~mm}$. Note that an adjustment of the stretch stub length $w_{4}$ is essential in controlling the transmission-zero frequency near the upper passband edge.

As shown in Fig. 11(a), an additional upper transmission zero would be observed at the higher frequency. When the stretch stub length $w_{4}$ increases, the upper transmission zero would move to the lower frequency, accompanied by a slight increase of cross-coupled capacitance $C_{2}$ to make the lower transmission zero slightly move to the high frequency. The curves to depict

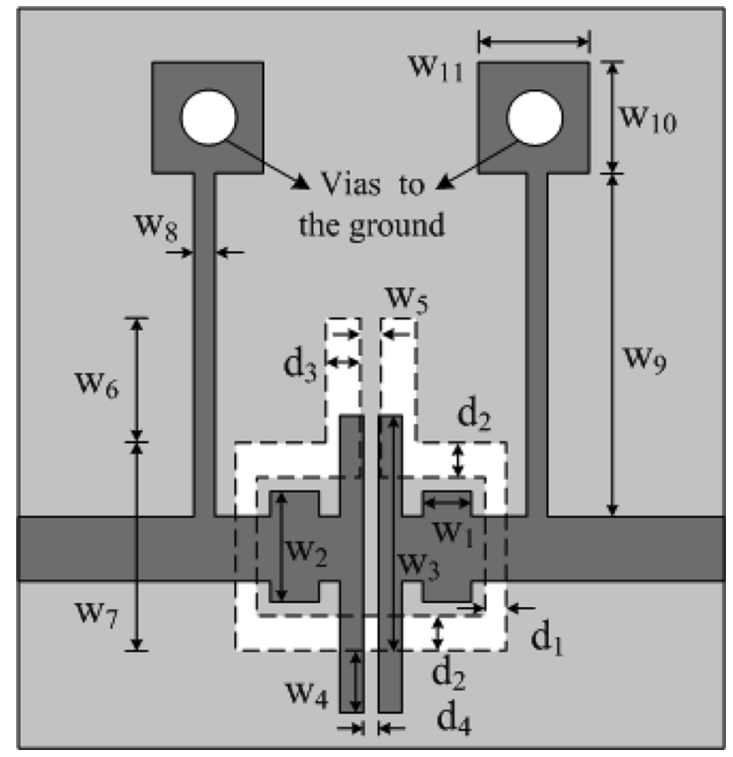

Fig. 10. Top-/bottom-layer circuit layouts of proposed five-pole UWB bandpass filter. $\left(w_{1}=0.89 \mathrm{~mm}, w_{2}=2.03 \mathrm{~mm}, w_{3}=4.318 \mathrm{~mm}, w_{4}=\right.$ $1.143 \mathrm{~mm}, w_{5}=0.38 \mathrm{~mm}, w_{6}=2.29 \mathrm{~mm}, w_{7}=3.81 \mathrm{~mm}, w_{8}=0.38 \mathrm{~mm}$, $w_{9}=6.22 \mathrm{~mm}, w_{10}=2.03 \mathrm{~mm}, w_{11}=2.03 \mathrm{~mm}, d_{1}=0.38 \mathrm{~mm}$, $d_{2}=0.635 \mathrm{~mm}, d_{3}=0.635 \mathrm{~mm}$, and $d_{4}=0.28 \mathrm{~mm}$ ).

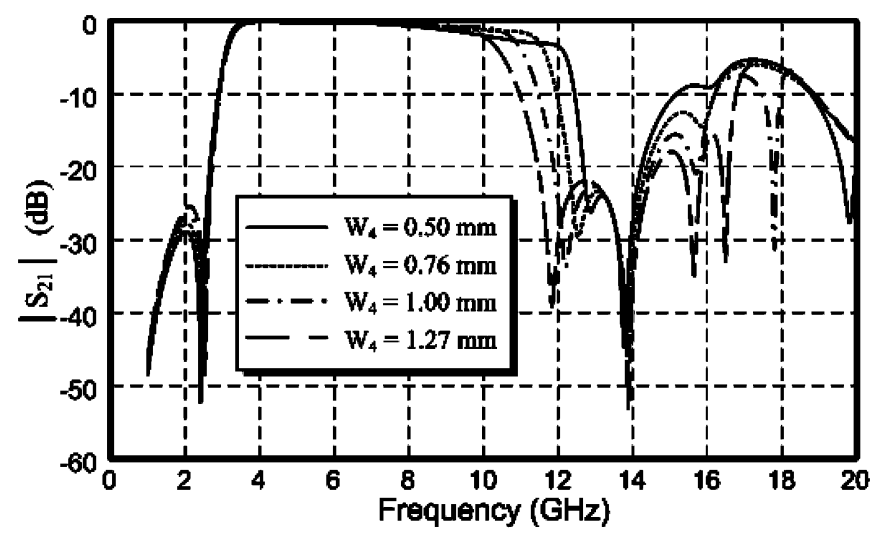

(a)

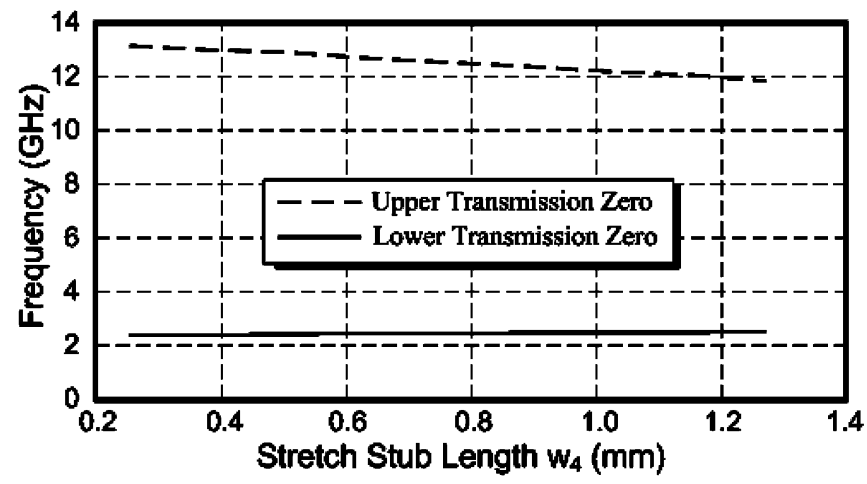

(b)

Fig. 11. (a) Simulated results of the proposed five-pole UWB filter (Fig. 10) for various values of the stretch stub length $w_{4}$. (b) Curves to relate the transmission-zero frequencies to the values of the stretch stub length $w_{4}$.

the relation between two transmission zeros and the stretch stub length $w_{4}$ are shown in Fig. 11(b), where the upper transmission zero decreases almost linearly and the lower transmission 


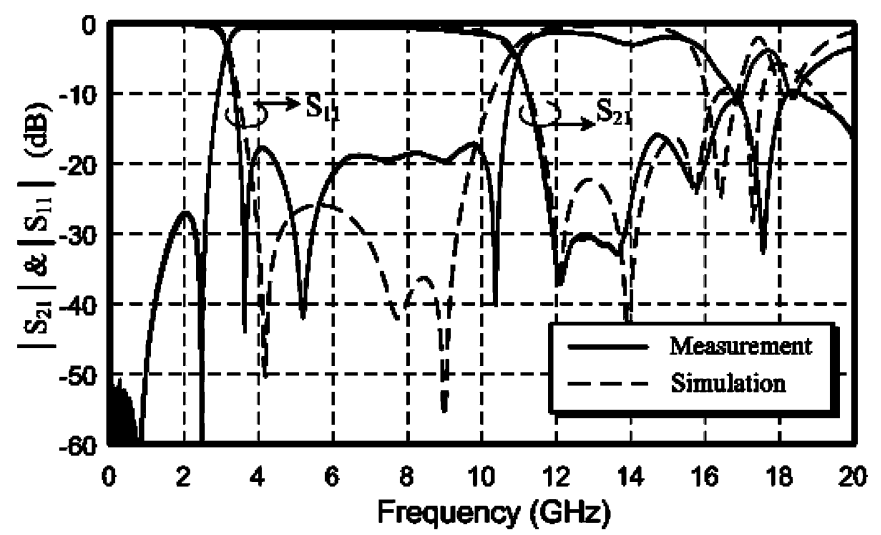

(a)

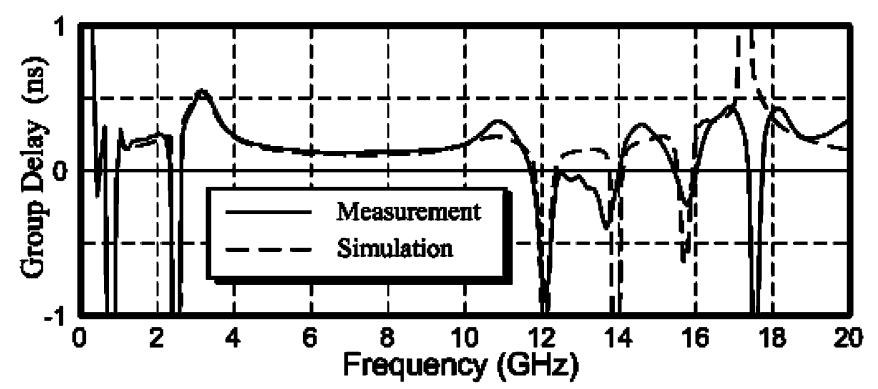

(b)

Fig. 12. Measured and simulated results of the proposed five-pole UWB filter (Fig. 10). (a) Scattering parameters. (b) Group delay.

zero increases slightly with the increasing stretch stub length $w_{4}$. After suitably designing the transition stretch stubs to the filter (Fig. 10), a five-pole UWB bandpass filter is proposed.

The measured and full-wave simulated responses of the fivepole UWB bandpass filter (Fig. 10) are shown in Fig. 12(a) with the filter again fabricated on the Rogers RO4003C substrate. The measured center frequency is at $6.95 \mathrm{GHz}$ and the measured $3-\mathrm{dB}$ fractional bandwidth is $108.5 \%$ from 3.18 to $10.72 \mathrm{GHz}$. This implemented filter has a minimum loss of $0.48 \mathrm{~dB}$, and the return loss is greater than $17.2 \mathrm{~dB}$ within the passband. Three transmission zeros are found at $2.45,12.11$, and $13.63 \mathrm{GHz}$. The first and second transmission zeros are generated by the cross-coupled capacitance and stretch stubs, respectively. Note that the third transmission zero is produced due to the resonance of the microstrip shorted stubs. With these transmission zeros, the five-order UWB bandpass filter has good selectivity and stopband rejection. Moreover, the implemented filter with the feeding lines deembedded exhibits flat group-delay response below 0.55 ns over the whole passband, as shown in Fig. 12(b). As in the case of the three-pole UWB bandpass filter, the group delay also exhibits the negative numbers around the frequencies of the transmission zeros. Besides, the proposed five-pole UWB bandpass filter has a very compact size of $8 \mathrm{~mm} \times 11.9 \mathrm{~mm}$, which is approximately $0.306 \lambda \times 0.458 \lambda$ at the center frequency $(=6.95 \mathrm{GHz})$.

\section{CONCLUSION}

In this paper, three- and five-pole UWB bandpass filters based on the microstrip-to-CPW transitions and CPW/microstrip shorted stubs connected to the ground have been implemented and carefully examined. By introducing a cross-coupled capacitance to the high-pass filter prototype and suitably designing the transition stretch stubs, the lower and upper transmission zeros have been generated and properly located. In the five-pole UWB bandpass filter, two microstrip shorted stubs used to realize two shunt inductances would resonate at higher frequency, thereby producing the third transmission zero at this higher frequency. With these transmission zeros, the proposed filters have good selectivity and stopband rejection. In addition, the dimensions of proposed UWB bandpass filters are much less than those of the published UWB filters. The proposed filters with good frequency performance and flat group delay are attractive for UWB radio applications.

\section{ACKNOWLEDGMENT}

The authors would like to thank Y.-S. Lin, National Central University, Chungli, Taiwan, R.O.C., for his helpful discussions.

\section{REFERENCES}

[1] "Revision of part 15 of the Commission's rules regarding ultra-wideband transmission systems," FCC, Washington, DC, Tech. Rep, ET-Docket 98-153, FCC02-48, adopted in Feb. 2002.

[2] J. S. Hong and M. J. Lancaster, Microstrip Bandpass Filters for RF/Microwave Applications. New York: Wiley, 2001

[3] Y. S. Lin, W. C. Ku, C. H. Wang, and C. H. Chen, "Wideband coplanar-waveguide bandpass filters with good stopband rejection," IEEE Microw. Wireless Compon. Lett., vol. 14, no. 9, pp. 422-424, Sep. 2004

[4] C. L. Hsu, F. C. Hsu, and J. T. Kuo, "Microstrip bandpass filter for ultra-wideband (UWB) wireless communications," in IEEE MTT-S Int. Microw. Symp. Dig., 2005, pp. 679-682.

[5] W. T. Wong, Y. S. Lin, C. H. Wang, and C. H. Chen, "Highly selective microstrip bandpass filters for ultra-wideband (UWB) applications," in Proc. Asia-Pacific Microw. Conf., Nov. 2005, pp. 2850-2853.

[6] H. Ishida and K. Araki, "Design and analysis of UWB bandpass filter with ring filter,' in IEEE MTT-S Int. Microw. Symp. Dig., Jun. 2004, pp. 1307-1310.

[7] W. Menzel, L. Zhu, K. Wu, and F. Bogelsack, "On the design of novel compact broadband planar filters," IEEE Trans. Microw. Theory Tech., vol. 51, no. 2, pp. 364-369, Feb. 2003.

[8] L. Zhu, S. Sun, and W. Menzel, "Ultra-wideband (UWB) bandpass filters using multiple-mode resonator," IEEE Microw. Wireless Compon. Lett., vol. 15, no. 11, pp. 796-798, Nov. 2005.

[9] L. Zhu, H. Bu, and K. Wu, "Aperture compensation technique for innovative design of ultra-broadband microstrip bandpass filter," in IEEE MTT-S Int. Microw. Symp. Dig., 2000, pp. 315-318.

[10] J. T. Kuo and E. Shih, "Wideband bandpass filter design with three-line microstrip structures," in IEEE MTT-S Int. Microw. Symp. Dig., 2001, pp. $1593-1596$.

[11] R. W. Jackson and D. W. Matolak, "Surface-to-surface transition via electromagnetic coupling of coplanar waveguide," IEEE Trans. Microw. Theory Tech., vol. MTT-35, no. 11, pp. 1027-1032, Nov. 1987.

[12] J. J. Burke and R. W. Jackson, "Surface-to-surface transition via electromagnetic coupling of microstrip and coplanar waveguide," IEEE Trans. Microw. Theory Tech., vol. 37, no. 3, pp. 519-525, Mar. 1989.

[13] L. Zhu and W. Menzel, "Broad-band microstrip-to-CPW transition via frequency-dependent electromagnetic coupling," IEEE Trans. Microw. Theory Tech., vol. 52, no. 5, pp. 1517-1522, May 2004.

[14] H. Wang, L. Zhu, and W. Menzel, "Ultra-wideband bandpass filters with hybrid microstrip/CPW structure," IEEE Microw. Wireless Compon. Lett., vol. 15, no. 12, pp. 844-846, Dec. 2005.

[15] K. Li, D. Kurita, and T. Matsui, "An ultra-wideband bandpass filter using broadside-coupled microstrip-coplanar waveguide structure," in IEEE MTT-S Int. Microw. Symp. Dig., 2005, pp. 675-678.

[16] D. M. Pozar, Microwave Engineering, 2nd ed. New York: Wiley, 1998 , ch. 8 . 


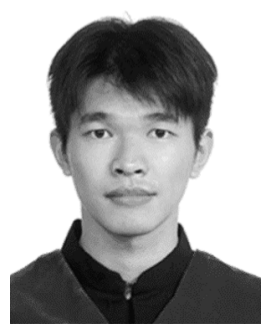

Tsung-Nan Kuo was born in Taoyuan, Taiwan, R.O.C., in 1981. He received the B.S. degree in electrical engineering from National Dong Hwa University, Hualien, Taiwan, R.O.C., in 2003, the M.S.E.E. degree from National Taiwan University, Taipei, Taiwan, R.O.C., in 2005, and is currently working toward the Ph.D. degree at National Taiwan University.

His research interests include the design and analysis of microwave filter circuits.

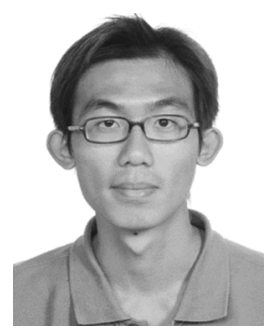

Shih-Cheng Lin was born in Taitung, Taiwan, R.O.C., in 1981. He received the B.S. degree in electrical engineering from National Sun Yet-Sen University, Kaohsiung, Taiwan, R.O.C., in 2003, and is currently working toward the Ph.D. degree in communication engineering at National Taiwan University, Taipei, Taiwan.

His research interests include the design and analysis of microwave filter circuits.

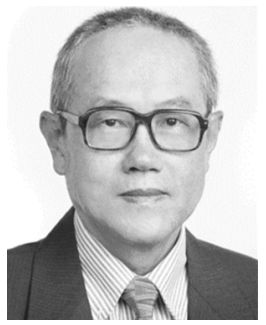

Chun Hsiung Chen (SM'88-F'96) was born in Taipei, Taiwan, R.O.C., on March 7, 1937. He received the B.S.E.E. and Ph.D. degrees in electrical engineering from National Taiwan University, Taipei, Taiwan, R.O.C., in 1960 and 1972, respectively, and the M.S.E.E. degree from National Chiao Tung University, Hsinchu, Taiwan, R.O.C., in 1962.

In 1963, he joined the Faculty of the Department of Electrical Engineering, National Taiwan University, where he is currently a Professor. From August 1982 to July 1985, he was Chairman of the Department of Electrical Engineering, National Taiwan University. From August 1992 to July 1996, he was the Director of the University Computer Center, National Taiwan University. In 1974, he was a Visiting Scholar with the Department of Electrical Engineering and Computer Sciences, University of California at Berkeley. From August 1986 to July 1987, he was a Visiting Professor with the Department of Electrical Engineering, University of Houston, Houston, TX. In 1989, 1990, and 1994, he visited the Microwave Department, Technical University of Munich, Munich, Germany, the Laboratoire d'Optique Electromagnetique, Faculte des Sciences et Techniques de Saint-Jerome, Universite d'Aix-Marseille III, Marseille, France, and the Department of Electrical Engineering, Michigan State University, East Lansing, respectively. His areas of interest include microwave circuits and computational electromagnetics. 